\title{
The role of resonances in the evolution of galactic disks
}

\author{
Jacques Lepine ${ }^{1}$, Sergio Scarano Jr. ${ }^{1}$, Sergei Andrievsky ${ }^{2}$, \\ Douglas A. de Barros ${ }^{1}$ and Thiago C. Junqueira ${ }^{1}$ \\ ${ }^{1}$ University of Sao Paulo Brazil, email: jacques@astro.iag.usp.br \\ ${ }^{2}$ Odessa National University
}

\begin{abstract}
Resonances play an important role in the evolution of the disks of spiral galaxies, and in particular in the chemical abundance evolution. The dominant effect is that of corotation; this effect can be even used as a tool to estimate the age of the present spiral arm pattern, which are usually found to be long-lived, contrary to a recent common belief. We investigated a sample of galaxies for which the corotation radius is known and for which there are available in the literature measurements of abundance gradients for Oxygen. A very good correlation is found between corotation radii and the radii at which there is a break in the slope of the gradients. The gradients are usually decreasing in the inner regions and become flat or rising at larger radii. In several galaxies, including the Milky Way, one observes not only a change in the slope of the abundance gradient, but also an abrupt step in metallicity, at corotation. This step is due to the fact that corotation separates the disk of a galaxy in two regions (inside corotation and outside corotation) which are isolated one from the other, so that the two sides evolve in an independent way. The barrier between the two regions is produced by the flow of gas in opposite directions in the two sides and by the ring-shaped void of gas observed at corotation. Besides this, an independent effect of corotation is a minimum of star formation associated with the minimum velocity at which the spiral arms (seen as potential wells) are fed with interstellar gas. Still another effect is the scattering of stars by the resonance, which causes their migration to different galactic radii. Other resonances, like 4:1, have properties almost opposite to corotation; they stimulate star-formation, and tend to gather the stars in the resonant orbit, instead of scattering them out, as shown by numerical simulations. Due to this property, one can see arms which have the shape of resonant stellar orbits, which depart from logarithmic spirals.
\end{abstract}

Keywords. galaxies: evolution; galaxies: structure 